\title{
Analytic expressions for the single particle energies with a quadrupole-quadrupole interaction and the relation to Elliott's $\mathrm{SU}(3)$ model
}

\author{
E. Moya de Guerra, ${ }^{1}$ P. Sarriguren, ${ }^{1}$ and L. Zamick ${ }^{2}$ \\ ${ }^{1}$ Instituto de Estructura de la Materia, Consejo Superior de Investigaciones Científicas, Serrano 123, E-28006 Madrid, Spain \\ ${ }^{2}$ Department of Physics and Astronomy, Rutgers University, Piscataway, New Jersey 08855
}

(Received 25 March 1997)

\begin{abstract}
We present an analytical proof and a generalization of the Fayache-Sharon-Zamick relation between single particle energy splittings and the SU(3) limit in Elliott's model. [S0556-2813(97)07308-1]
\end{abstract}

PACS number(s): 21.60.Cs, 21.60.Fw, 21.10.Pc

\section{INTRODUCTION}

In a recent publication [1], Fayache, Sharon, and Zamick compared the collective model result for a rotational band to the $0 d-1 s$ splitting obtained with the OXBASH program [2]. For the shell model calculations done with OXBASH they use a Hamiltonian consisting of a spherical harmonic oscillator and a residual interaction which is the isoscalar quadrupolequadrupole $(Q-Q)$ in coordinate space only, i.e., they omit the $Q \cdot Q$ term in momentum space and allow for interactions between shells with $\Delta N=2$. From these shell model calculations the authors of Ref. [1] found that one third of the $0 d-1 s$ single particle (s.p.) splitting $(6 \bar{\chi})$ comes from the interaction of the valence particle with the core while twothirds $(12 \bar{\chi})$ come from the diagonal $Q \cdot Q$ interaction. The $0 d-1 s$ s.p. energy-splitting $(18 \bar{\chi})$ is the same as the energy splitting between $L=2$ and $L=0$ rotational bands obtained with Elliott's SU(3) model in the $s-d$ shell [3]. A similar numerical relation was found in the $f-p$ shell [1]. This is an interesting observation that deserves further study.

In this paper we give an analytical proof of those numerical relations and show that they are particular cases of a general property of the Hamiltonian used. To this end we first derive complete analytical expressions for the s.p. energies (these will be defined more precisely in Sec. II), and then derive the s.p. energy splittings in a major shell using the same Hamiltonian as in Ref. [1]. We consider the problem of how to obtain the s.p. energy splittings in a major shell with such an interaction which will preserve the SU(3) results.

More specifically, we use the Hamiltonian

$$
H=\frac{\hbar \omega_{0}}{2} \sum_{i}\left(\mathbf{x}_{i}^{2}+\boldsymbol{\pi}_{i}^{2}\right)-\frac{\chi}{2} \sum_{i j} Q(i) \cdot Q(j),
$$

where

$$
\begin{gathered}
Q(i) \cdot Q(j)=\sum_{\mu} Q_{\mu}(i) Q_{\mu}^{\dagger}(j), \\
Q_{\mu}(i)=b^{2} x_{i}^{2} Y_{2}^{\mu}\left(\hat{x}_{i}\right),
\end{gathered}
$$

with dimensionless coordinate and momenta $\mathbf{x}_{i}=\mathbf{r}_{i} / b, \boldsymbol{\pi}_{i}$ $=\mathbf{p}_{i} b / \hbar$, and the harmonic oscillator (H.O.) length scale $b$ $=\sqrt{\hbar / m \omega_{0}}$.

\section{DEFINITION OF SINGLE PARTICLE ENERGIES AND ENERGY SPLITTINGS}

To parallel the shell model calculations of Ref. [1], we consider the effect of the residual $Q-Q$ interaction when we have a closed core and put a valence nucleon in different orbital $(N \ell \mathrm{m})$ states of a major shell $(N)$ out of the closed core. In other words, we look for the s.p. energy splitting between $N \ell$ and $N \ell^{\prime}$ levels caused by the $Q-Q$ interaction. To this end we write the s.p. energy of the $N \ell$ level as

$$
E_{N \ell}=\hbar \omega_{0}\left(N+\frac{3}{2}\right)+E_{N \ell}^{Q 1}+E_{N \ell}^{Q 2},
$$

where $E_{N \ell}^{Q 1}$ comes from the diagonal part of the $Q-Q$ interaction $(Q(i) \cdot Q(i))$

$$
E_{N \ell}^{Q 1}=-\frac{\chi}{2}\langle N \ell|Q \cdot Q| N \ell\rangle
$$

or

$$
E_{N \ell}^{Q 1}=-4 \bar{\chi}\left\langle N \ell\left|x^{4}\right| N \ell\right\rangle, \quad \bar{\chi}=\chi \frac{5 b^{4}}{32 \pi},
$$

while $E_{N \ell}^{Q 2}$ comes from the interaction of the valence particle with the core. The direct term of the two-body interaction $(Q(i) . Q(j), i \neq j)$ is proportional to the quadrupole moment of the core, hence it is zero for a closed core. Consequently $E_{N \ell}^{Q 2}$ is given by the exchange term

$$
E_{N \ell}^{Q 2}=\chi_{N_{c} \ell_{c} m_{c} \mu}\left|\left\langle N \ell m\left|Q_{\mu}\right| N_{c} \ell_{c} m_{c}\right\rangle\right|^{2} .
$$

Clearly the s.p. energy defined in Eq. (4) corresponds to the energy difference of the systems with $A+1$ and $A$ (core) nucleons, calculated as the expectation values of the Hamiltonian (1) in the ground state of the unperturbed $(\chi=0)$ harmonic oscillator Hamiltonian, i.e., in the simplest, lower order harmonic oscillator shell model wave function. We adopt this definition to follow the numerical work described in Ref. [1], although this is not the most general definition of s.p. energy that may be considered with the Hamiltonian (1).

Using the explicit expression for H.O. wave functions in terms of Laguerre polynomials we obtain the following result for $E_{N \ell}^{Q 1}$ : 


$$
\begin{aligned}
E_{N \ell}^{Q 1}= & -4 \bar{\chi}\left\{n(n-1)+4 n\left(n+\ell+\frac{3}{2}\right)\right. \\
& \left.+\left(n+\ell+\frac{3}{2}\right)\left(n+\ell+\frac{5}{2}\right)\right\} \\
= & \bar{\chi}\left[2 \ell(\ell+1)-3\left(2 N^{2}+6 N+5\right)\right],
\end{aligned}
$$

with $n$ the principal quantum number $(n=(N-\ell) / 2)$ (see, for instance, Ref. [4]).

To evaluate Eq. (7) we have to keep in mind that according to the selection rules for H.O. wave functions only matrix elements with $N_{c}=N-2$ will contribute to the sum over $N_{c}$. This is clear since the $Q_{\mu}$ operator only connects the states with $N$ to those with $N^{\prime}=N, N \pm 2$, and of these possible $N^{\prime}$ values only $N^{\prime}=N_{c}=N-2$ belongs to the set of core levels. Carrying out the sum over $m_{c}$ and $\mu$ in Eq. (7) we get

$$
\begin{aligned}
E_{N \ell}^{Q 2}= & 8 \bar{\chi} \sum_{\ell_{c}}\left(\left\langle N \ell\left|x^{2}\right| N-2 \ell_{c}\right\rangle\right)^{2}\left(2 \ell_{c}+1\right) \\
& \times\left(\begin{array}{ccc}
\ell & 2 & \ell_{c} \\
0 & 0 & 0
\end{array}\right)^{2} .
\end{aligned}
$$

For H.O. wave functions the matrix element in Eq. (9) is given by

$$
\begin{aligned}
\langle N \ell & \left.\left|x^{2}\right| N-2 \ell^{\prime}\right\rangle \\
= & \delta_{\ell^{\prime}, \ell} \sqrt{n\left(n+\ell+\frac{1}{2}\right)}+\delta_{\ell^{\prime}, \ell+2} \sqrt{n(n-1)} \\
& +\delta_{\ell^{\prime}, \ell-2} \sqrt{\left(n+\ell+\frac{1}{2}\right)\left(n+\ell-\frac{1}{2}\right)}
\end{aligned}
$$

Substitution of Eq. (10) into Eq. (9), together with the following equalities that hold for $3-j$ symbols:

$$
\begin{aligned}
& \sum_{\ell^{\prime}}\left(2 \ell^{\prime}+1\right)\left(\begin{array}{lll}
\ell & 2 & \ell^{\prime} \\
0 & 0 & 0
\end{array}\right)^{2}=1, \\
& \frac{(2 \ell+3)}{2}\left(\begin{array}{lll}
\ell & 2 & \ell \\
0 & 0 & 0
\end{array}\right)^{2}+(2 \ell-3)\left(\begin{array}{ccc}
\ell & 2 & \ell-2 \\
0 & 0 & 0
\end{array}\right)^{2} \\
& =\frac{\ell}{2 \ell+1}, \\
& \quad(2 \ell-1)(2 \ell-3)\left(\begin{array}{ccc}
\ell & 2 & \ell-2 \\
0 & 0 & 0
\end{array}\right)^{2}=\frac{3}{2} \frac{\ell(\ell-1)}{(2 \ell+1)},
\end{aligned}
$$

leads to the following result for the valence core interaction:

$$
\begin{aligned}
E_{N \ell}^{Q 2} & =8 \bar{\chi}\left\{n(n-1)+n \ell+\frac{3}{8} \ell(\ell-1)\right\} \\
& =\bar{\chi}[\ell(\ell+1)+2 N(N-2)] .
\end{aligned}
$$

It is now a simple matter to compute the energy splitting $\Delta_{N\left(\ell, \ell^{\prime}\right)}$ between $N$-shell orbital partners $N \ell$ and $N \ell^{\prime}$ :

$$
\Delta_{N\left(\ell, \ell^{\prime}\right)} \equiv\left(E_{N \ell}-E_{N \ell^{\prime}}\right)=3 \bar{\chi}\left\{\ell(\ell+1)-\ell^{\prime}\left(\ell^{\prime}+1\right)\right\},
$$

or for $\ell^{\prime}=\ell-2$

$$
\Delta_{N\left(\ell, \ell^{\prime}=\ell-2\right)}=6 \bar{\chi}(2 \ell-1),
$$

which agree with the $\mathrm{SU}(3)$ result. In particular in the $N$ $=2$ and $N=3$ shells we recover the numerical results of Ref. [1].

We can also show easily that one-third of the energy splitting $\Delta_{N\left(\ell, \ell^{\prime}\right)}$ comes from the valence-core interaction and two-thirds from the diagonal term of $Q-Q$. Writing

$$
\Delta_{N\left(\ell, \ell^{\prime}\right)}=\Delta_{N\left(\ell, \ell^{\prime}\right)}^{Q 1}+\Delta_{N\left(\ell, \ell^{\prime}\right)}^{Q 2}
$$

with

$$
\begin{aligned}
& \Delta_{N\left(\ell, \ell^{\prime}\right)}^{Q 1} \equiv E_{N \ell}^{Q 1}-E_{N \ell^{\prime},}^{Q 1}, \\
& \Delta_{N\left(\ell, \ell^{\prime}\right)}^{Q 2} \equiv E_{N \ell}^{Q 2}-E_{N \ell^{\prime},}^{Q 2},
\end{aligned}
$$

we obtain using Eqs. (8) and (14)

$$
\begin{aligned}
& \Delta_{N\left(\ell, \ell^{\prime}\right)}^{Q 1}=2 \bar{\chi}\left[\ell(\ell+1)-\ell^{\prime}\left(\ell^{\prime}+1\right)\right], \\
& \Delta_{N\left(\ell, \ell^{\prime}\right)}^{Q 2}=\bar{\chi}\left[\ell(\ell+1)-\ell^{\prime}\left(\ell^{\prime}+1\right)\right],
\end{aligned}
$$

or

$$
\begin{aligned}
& \left.\begin{array}{l}
\Delta_{N\left(\ell, \ell^{\prime}\right)}^{Q 1}=\frac{2}{3} \Delta_{N\left(\ell, \ell^{\prime}\right)} \\
\Delta_{N\left(\ell, \ell^{\prime}\right)}^{Q 2}=\frac{1}{3} \Delta_{N\left(\ell, \ell^{\prime}\right)}
\end{array}\right\} \\
& =\left\{\begin{array}{ll}
4(2 \ell-1) \bar{\chi} & \text { for } \quad \ell^{\prime}=\ell-2 \\
2(2 \ell-1) \bar{\chi} & \text { for } \quad \ell^{\prime}=\ell-2
\end{array}\right\},
\end{aligned}
$$

which again particularize to the numerical results found in Ref. [1] for the $N=2(1 s-0 d)$ and the $N=3(1 p-0 f)$ shells

$$
\begin{aligned}
\Delta_{2(2,0)}^{Q 1}=12 \bar{\chi}, & \Delta_{3(3,1)}^{Q 1}=20 \bar{\chi}, \\
\Delta_{2(2,0)}^{Q 2}=6 \bar{\chi}, & \Delta_{3(3,1)}^{Q 2}=10 \bar{\chi}, \\
\Delta_{2(2,0)}=18 \bar{\chi}, & \Delta_{3(3,1)}=30 \bar{\chi} .
\end{aligned}
$$

We note that although not explicitly mentioned, we have been considering the valence particle in a shell close to the core. In principle we may as well consider a valence particle in a higher $N$-shell, $N_{v}>N_{c}^{\max }+2$. In that case the valencecore interaction is zero and only the $Q 1$ splitting remains, i.e., for these higher $N$-shells one gets a smaller splitting.

\section{COMPARISON WITH ELLIOTT'S $Q \cdot Q$ INTERACTION}

One may wonder how the one-third, two-thirds division of the s.p. energy splitting between core-particle and diagonal contributions might be related to the inclusion of the momentum-dependent parts of the Elliott quadrupole operator $Q_{\mu}^{E}$, 
TABLE I. Reduced matrix elements of quadrupole operators in $r$ and $p$ spaces. Note that $\left\langle N \ell^{\prime}\left\|q_{p}\right\| N \ell\right\rangle=\left\langle N \ell^{\prime}\left\|q_{r}\right\| N \ell\right\rangle$, $\left\langle(N \pm 2) \ell^{\prime}\left\|q_{p}\right\| N \ell\right\rangle=-\left\langle(N \pm 2) \ell^{\prime}\left\|q_{r}\right\| N \ell\right\rangle$.

\begin{tabular}{|c|c|c|c|}
\hline & $\left\langle N \ell^{\prime}\left\|q_{p}\right\| N \ell\right\rangle$ & $\left\langle(N-2) \ell^{\prime}\left\|q_{p}\right\| N \ell\right\rangle$ & $\left\langle(N+2) \ell^{\prime}\left\|q_{p}\right\| N \ell\right\rangle$ \\
\hline \multirow{2}{*}{$\ell^{\prime}=\ell$} & $(3) \sqrt{\ell(\ell+1)(2 \ell+1)}$ & $\sqrt{n(n+\ell+1 / 2) \ell(\ell+1)(2 \ell+1)}$ & $\sqrt{(n+1)(n+\ell+3 / 2) \ell(\ell+1)(2 \ell+1)}$ \\
\hline & $-\left(N+\frac{-}{2}\right) \sqrt{(2 \ell-1)(2 \ell+3)}$ & $\sqrt{(2 \ell-1)(2 \ell+3)}$ & $\sqrt{(2 \ell-1)(2 \ell+3)}$ \\
\hline \multirow{2}{*}{$\ell^{\prime}=\ell-2$} & $\sqrt{6(n+1)(n+\ell+1 / 2) \ell(\ell-1)}$ & $\sqrt{3(n+\ell+1 / 2)(n+\ell-1 / 2) \ell(\ell-1)}$ & $\sqrt{3(n+1)(n+2) \ell(\ell-1)}$ \\
\hline & $\sqrt{6}-2 \ell-1$ & $-\sqrt{2 \quad 2 \ell-1}$ & $-\sqrt{2 \quad 2 \ell-1}$ \\
\hline \multirow{2}{*}{$\ell^{\prime}=\ell+2$} & $\sqrt{n(n+\ell+3 / 2)(\ell+1)(\ell+2)}$ & $\sqrt{3 n(n-1)(\ell+1)(\ell+2)}$ & $3(n+\ell+5 / 2)(n+\ell+3 / 2)(\ell+1)(\ell+2)$ \\
\hline & $\sqrt{6} \frac{2 \ell+3}{2 \ell}$ & $-\sqrt{2 \quad 2 \ell+3}$ & $2 \ell+3$ \\
\hline
\end{tabular}

$$
\begin{aligned}
Q_{\mu}^{E}(i) & =\frac{1}{2} b^{2}\left[x_{i}^{2} Y_{2}^{\mu}\left(\hat{x}_{i}\right)+\pi_{i}^{2} Y_{2}^{\mu}\left(\hat{\pi}_{i}\right)\right] \\
& \equiv \frac{1}{2} b^{2} \sqrt{\frac{5}{4 \pi}}\left(q_{r_{i}}^{\mu}+q_{p_{i}}^{\mu}\right) .
\end{aligned}
$$

It is therefore instructive to study how the s.p. energy splitting is shared by the position, momentum, and crossed terms of the Elliott $Q \cdot Q$ interaction

$$
H_{Q Q}^{E}=-\frac{\chi}{2} \sum_{i j} Q^{E}(i) \cdot Q^{E}(j)
$$

We stress that this interaction is formally identical to that in Eq. (1). The only difference comes from the replacement of the position quadrupole operator $Q$ by the $Q^{E}$ operator. The latter is a sum of the dimensionless position and momentum quadrupole operators

$$
\begin{aligned}
q_{r}^{\mu} & =\sqrt{\frac{4 \pi}{5}} x_{i}^{2} Y_{2}^{\mu}\left(\hat{x}_{i}\right), \\
q_{p}^{\mu} & =\sqrt{\frac{4 \pi}{5}} \pi_{i}^{2} Y_{2}^{\mu}\left(\hat{\pi}_{i}\right) .
\end{aligned}
$$

We recall that an important property of Elliott's quadrupole operator is that it has zero matrix elements between different $N$-shell states because the $\Delta N=2$ matrix elements of $q_{p}$ exactly cancel those of $q_{r}$. As can be seen from Table I the reduced matrix elements of $Q_{\mu}^{E}$ are

$$
\begin{aligned}
\left\langle N^{\prime} \ell^{\prime}\left\|Q_{\mu}^{E}\right\| N \ell\right\rangle & =\delta_{N N^{\prime}} \sqrt{\frac{5}{4 \pi}} b^{2}\left\{-\delta_{\ell, \ell^{\prime}}\left(N+\frac{3}{2}\right) \sqrt{\frac{\ell(\ell+1)(2 \ell+1)}{(2 \ell-1)(2 \ell+3)}}\right. \\
& +\delta_{\ell^{\prime}, \ell-2} \sqrt{\frac{3}{2} \frac{(N-\ell+2)(N+\ell+1) \ell(\ell-1)}{2 \ell-1}} \\
& \left.+\delta_{\ell^{\prime}, \ell+2} \sqrt{\frac{3}{2} \frac{(N-\ell)(N+\ell+3)(\ell+1)(\ell+2)}{2 \ell+3}}\right\} .
\end{aligned}
$$

Therefore, with Elliott's interaction there is no particle-core interaction and the s.p. energy defined in the previous section
[Eq. (4)], only gets a contribution from the diagonal term defined in Eq. (5) with $Q$ replaced by $Q^{E}$. This diagonal contribution can be easily calculated from Eq. (28), and it is found to be

$$
E_{N \ell}^{Q E}=\bar{\chi}[3 \ell(\ell+1)-4 N(N+3)] .
$$

Hence, when $Q$ is replaced by $Q^{E}$ in Eq. (1), the sum of the one-body $\left(E_{N \ell}^{Q 1}\right)$ and two-body $\left(E_{N \ell}^{Q 2}\right)$ contributions to the s.p. energy [Eq. (4)] is replaced by the one-body contribution $E_{N \ell}^{Q E}$. The resulting value of the s.p. energy differs only in the $N$-dependence and therefore one is left with identical s.p. energy splittings:

$$
\begin{aligned}
\Delta_{N\left(\ell, \ell^{\prime}\right)}^{E} & =E_{N \ell}^{Q E}-E_{N \ell^{\prime}}^{Q E} \\
& =3 \bar{\chi}\left[\ell(\ell+1)-\ell^{\prime}\left(\ell^{\prime}+1\right)\right] \equiv \Delta_{N\left(\ell, \ell^{\prime}\right)} .
\end{aligned}
$$

We also note that this s.p. energy splitting can be decomposed in three contributions,

$$
\Delta_{N\left(\ell, \ell^{\prime}\right)}^{E}=\Delta_{N\left(\ell, \ell^{\prime}\right)}^{E_{r}}+\Delta_{N\left(\ell, \ell^{\prime}\right)}^{E_{p}}+\Delta_{N\left(\ell, \ell^{\prime}\right)}^{E_{r p}}
$$

one coming from the $q_{r} \cdot q_{r}$ interaction $\left(\Delta^{E_{r}}\right)$, one coming from the $q_{p} \cdot q_{p}$ interaction $\left(\Delta^{E_{p}}\right)$, and one coming from the crossed $q_{r} \cdot q_{p}+q_{p} \cdot q_{r}$ interaction $\left(\Delta^{E_{r p}}\right)$. Using Table I, it is straightforward to check that

$$
\begin{aligned}
& E_{N \ell}^{E_{r}} \equiv-\bar{\chi}\left\langle N \ell\left|q_{r} \cdot q_{r}\right| N \ell\right\rangle \\
&=\bar{\chi}\left[\frac{1}{2} \ell(\ell+1)-\frac{3}{4}\left(2 N^{2}+6 N+5\right)\right], \\
& E_{N \ell}^{E p} \equiv-\bar{\chi}\left\langle N \ell\left|q_{p} \cdot q_{p}\right| N \ell\right\rangle=E_{N \ell}^{E_{r}}, \\
& E_{N \ell}^{E_{r p}} \equiv-\bar{\chi}\left\langle N \ell\left|q_{r} \cdot q_{p}+q_{p} \cdot q_{r}\right| N \ell\right\rangle \\
&=\bar{\chi}\left[2 \ell(\ell+1)-\left(N^{2}+3 N-\frac{15}{2}\right)\right] .
\end{aligned}
$$

Therefore the SU(3) s.p. energy splitting $3 \bar{\chi}[\ell(\ell+1)$ $\left.-\ell^{\prime}\left(\ell^{\prime}+1\right)\right]$ is shared as one-sixth, one-sixth, and twothirds by the position, momentum, and crossed terms, respectively: 


$$
\begin{gathered}
\Delta_{N\left(\ell, \ell^{\prime}\right)}^{E_{r}}=\Delta_{N\left(\ell, \ell^{\prime}\right)}^{E_{p}}=\frac{1}{6} \Delta_{N\left(\ell, \ell^{\prime}\right)}, \\
\Delta_{N\left(\ell, \ell^{\prime}\right)}^{E_{r p}}=\frac{2}{3} \Delta_{N\left(\ell, \ell^{\prime}\right) .}
\end{gathered}
$$

\section{A NOTE ON SPIN-ORBIT INTERACTION}

Since spin-orbit coupling is very important in nuclei it is also useful to examine how the above results are affected when the one body spin-orbit interaction $\left(V_{S O}=\right.$ $-2 \chi_{s o} \mathbf{l}$.s), is added to the Hamiltonian. Different cases have to be distinguished. The simplest cases are the $s-d$ shell and $f$ - $p$ shell nuclei where the closed cores consist of closed $N$-shells $(N=Z=8$ or $N=Z=20)$. In these cases the results given in the previous section still hold for $N \ell$ and $N \ell^{\prime}$ orbitals. The only difference is that one may in addition consider the energy splitting between different $\ell j$ and $\ell^{\prime} j^{\prime}$ subshells. Since the energy splitting between $(j=\ell-1 / 2)$ and $(j=\ell+1 / 2)$ partners is not perturbed by the $Q-Q$ interaction, the total $N \ell j-N \ell^{\prime} j^{\prime}$ splittings are for $\ell^{\prime}=\ell-2$ :

$$
\begin{aligned}
& \Delta_{N\left(\ell j, \ell^{\prime} j^{\prime}\right)} \\
& \quad+6 \bar{\chi}(2 \ell-1) \\
& \quad\left(\chi_{s 0}\left\{\begin{array}{l}
\mp 2 \text { for } j=\ell \pm \frac{1}{2} \text { and } j^{\prime}=j-2 \\
-(2 \ell-1) \text { for } j^{\prime}=j-3\left(j=\ell+\frac{1}{2}\right) \\
+(2 \ell-1) \text { for } j^{\prime}=j-1\left(j=\ell-\frac{1}{2}\right)
\end{array}\right\},\right.
\end{aligned}
$$

i.e., the spin-orbit in general destroys the SU(3) limit result, but for some of the $\ell j-\ell^{\prime} j^{\prime}$ splittings the proportionality to $(2 \ell-1)$ remains.

On the other hand when the closed core corresponds to magic numbers with $N$ or $Z=28,50$, etc., where only one of the $N \ell j$ subshells of the valence shell $N$ (the $\ell j$ subshell with $n=0, \ell=N, j=\ell+1 / 2$ ) is closed, one has to take into account in addition the $Q-Q$ interaction of the valence particle with this subshell, i.e., when $N$ or $Z=28,50,82,126$ the two-body interaction contains also the term

$$
\delta E_{N \ell j}^{Q 2}=\chi \sum_{m_{j c} \mu}\left|\left\langle N \ell_{j m}\left|Q_{\mu}\right| N \ell_{c} j_{c} m_{j c}\right\rangle\right|^{2}
$$

with $\ell_{c}=N$ and $j_{c}=\ell_{c}+1 / 2=N+1 / 2$.

This contribution gives an extra term to the energy of the valence subshells with quantum numbers

$$
\text { (a) } \ell=\ell_{c}=N, \quad j=j_{c}-1=N-\frac{1}{2}
$$

and

$$
\text { (b) } \ell=\ell_{c}-2=N-2 ; j=\ell_{c}-2+\frac{1}{2}=N-\frac{3}{2} \text {. }
$$

The value of this extra term is (a) $\delta E_{N / j}^{Q 2}=6 \bar{\chi} \frac{(2 N+3)(N+1)}{(2 N+1)(2 N-1)} \rightarrow 3 \bar{\chi}$ for $N$ large,

(b) $\delta E_{N / j}^{Q 2}=24 \bar{\chi} \frac{N(N+1)}{2 N-1} \rightarrow 12 \bar{\chi} N$ for $N$ large.

Thus, this extra contribution may also spoil the SU(3) limit $[$ Eq. (15)] for some of the s.p. energy splittings.

\section{CONCLUSIONS}

In summary, quite generally, the s.p. energy splitting in a major shell $N$ preserving the $\mathrm{SU}(3)$ result

$$
\begin{aligned}
\Delta_{N\left(\ell, \ell^{\prime}\right)} & \equiv E_{N \ell}-E_{N \ell^{\prime}} \\
& =\Delta_{N\left(\ell, \ell^{\prime}\right)}^{Q 1}+\Delta_{N\left(\ell, \ell^{\prime}\right)}^{Q 2}=\Delta_{N\left(\ell, \ell^{\prime}\right)}^{E} \\
& =3 \bar{\chi}\left[\left\langle N \ell\left|\mathbf{L}^{2}\right| N \ell\right\rangle-\left\langle N \ell^{\prime}\left|\mathbf{L}^{2}\right| N \ell^{\prime}\right\rangle\right]
\end{aligned}
$$

has been obtained with a $Q-Q$ interaction in coordinate space that allows for interactions between $\Delta N=2$ shells. As a general rule, the valence-core interaction - proportional to the matrix elements of the $Q$-operator between $\Delta N=2$ shells generates one-third of the energy splitting in Eq. (41), while the other two-thirds are due to the diagonal (one-body) part of $Q \cdot Q$. This result is surprising since traditionally the SU(3) limit of Elliott's model was derived restricting the action of the $Q-Q$ interaction to a single major shell (eliminating the $\Delta N=2$ matrix elements of the $Q$ operator).

We have also explicitly shown that with Elliott quadrupole operator the same s.p. energy splitting is obtained by adding up the contributions from the position, momentum, and crossed position-momentum terms of the Elliott quadrupole-quadrupole interaction. This comparison is important because it illustrates how in this instance, the same effect can be obtained by taking into account (two-body) particle-hole interactions or (one-body) momentum dependent interactions. The fact that the s.p. energy splittings are equal while the s.p. energies are not, also serves to illustrate how the two types of interactions can be equivalent in some aspects while differing in others.

Our conclusion here generalizes and reinforces the observation made in Ref. [1] for the $N=2,3$ shells and suggests new ways for further applications of the model Hamiltonian (1). Since applications of this model Hamiltonian in the past have proven to be very fruitful it is worth while to explore it in a new direction. A clear practical application involves deriving the $Q \cdot Q$ interaction from the realistic interaction. One now does not have to justify the momentum terms.

We have also found that although the spin-orbit coupling tends to spoil the $\mathrm{SU}(3)$ limit in Eq. (41), the proportionality to $(2 \ell+3)$ of the s.p. energy splitting between $(\ell, j)$ and $\left(\ell+2, j^{\prime}\right)$ subshells is still maintained in some cases.

\section{ACKNOWLEDGMENTS}

This work was supported by DGICYT (Spain) under Contract No. PB95/0123 and by U.S. Department of Energy Grant No. DE-FG05-95ER-40940. One of us (L.Z.) acknowledges support from DGICYT (Spain) under Contract No. SA95-0371. 
[1] M. S. Fayache, Y. Sharon, and L. Zamick, Phys. Rev. C 55, 1575 (1997).

[2] B.A. Brown, A. Etchegoyen, and W. D. M. Rae, the computer code OXBASH, MSU-NSCL Report No. 524, 1992.

[3] J. P. Elliott, Proc. R. Soc. London, Ser. A 245, 128 (1958);
245, 562 (1958).

[4] I.S. Gradshteyn and I.M. Ryzhik, Table of Integrals, Series and Products (Academic, London 1980); P. Morse and H. Feshbach, Methods of Theoretical Physics (McGraw Hill, New York, 1953). 